\title{
The Extraction of the Nitrates of the Rare Earth Metals by Mixtures of Binary and Neutral Extractants
}

\author{
Sergey N. Kalyakin, \\ Marina A. Mulagaleeva* and Anna A. Kuzmina \\ Institute of Chemistry and Chemical Technology SB RAS \\ FRC "Krasnoyarsk Science Center SB RAS" \\ Krasnoyarsk, Russian Federation
}

Received 28.05.2020, received in revised form 04.06.2020, accepted 18.08.2020

\begin{abstract}
The extraction of the nitrates of the rare earth metals with mixtures consisting of binary extractants was explored: 2-ethylhexyl-mono-2-ethylhexyl ether phosphonate tri-n-octylamine, di(2-ethylhexyl) phosphate tri-n-octylamine and a neutral extractant (solvating reagent)-tri-n-octylamine nitrate. It was established that the extraction of the nitrates of the REM occurs in accordance with the patterns of binary extraction. Extraction systems are characterized by high selectivity in regard to rare-earth metals.
\end{abstract}

Keywords: REM, binary extraction, tri-n-octylamine, DEHPA, EHEHPA.

Citation: Kalyakin S.N., Mulagaleeva M.A., Kuzmina A.A. The extraction of the nitrates of the rare earth metals by mixtures of binary and neutral extractants, J. Sib. Fed. Univ. Chem., 2020, 13(3), 363-371. DOI: 10.17516/1998-2836-0189

(c) Siberian Federal University. All rights reserved

This work is licensed under a Creative Commons Attribution-NonCommercial 4.0 International License (CC BY-NC 4.0).

* Corresponding author E-mail address: maral7508@mail.ru 


\title{
Экстракция нитратов редкоземельных металлов смесями бинарных и нейтральных экстрагентов
}

\author{
С.Н. Калякин, М.А. Мулагалеева, А.А. Кузьмина \\ Институт химии и химической технологии \\ ФИЦ «Красноярский научный центтр СО РАН» \\ Российская Федерация, Красноярск
}

\begin{abstract}
Аннотация. Исследована экстракция нитратов редкоземельных металлов (РЗМ) смесями, состоящими из бинарных экстрагентов: 2-этилгексил-моно-2-этилгексил эфир фосфонат три-н-октиламина, ди-(2-этилгексил)фосфат три-н-октиламина и нейтрального экстрагена (сольватирующий реагент) - нитрат триоктиламина. Установлено, что извлечение нитратов РЗМ происходит в соответствии с закономерностями бинарной экстракции. Экстракционные системы характеризуются высокой селективностью по отношению к РЗМ.
\end{abstract}

Ключевые слова: РЗМ, бинарная экстракция, три-н-октиламин, Д2ЭГФК, ЕНЕНРА.

Цитирование: Калякин, С.Н. Экстракция нитратов редкоземельных металлов смесями бинарных и нейтральных экстрагентов / С.Н. Калякин, М.А. Мулагалеева, А.А. Кузьмина // Журн. Сиб. федер. ун-та. Химия, 2020. 13(3). С. 363371. DOI: $10.17516 / 1998-2836-0189$

\section{Введение}

Бинарные экстрагенты - это растворы в органических разбавителях солей, полученных из органических высокомолекулярных кислот и оснований [1-3]. Соответственно, бинарные экстрагенты могут быть приготовлены на основе стехиометрических смесей известных катионои анионообменных экстрагентов. В предыдущих исследованиях нами установлено [4-6], что наиболее высокую избирательность при экстракции солей лантаноидов проявляют бинарные экстрагенты, приготовленные на основе фосфорсодержащих кислот, таких как 2-этилгексилмоно-2-этилгексил эфир фосфоновой кислоты (EHEHРA, HA) и ди-(2-этилгексил)фосфорная кислота (DEHPA, HA). В качестве органического основания удобнее всего использовать триоктиламин (TOA, $\left.N R_{3}\right)$.

Один из существенных недостатков указанных бинарных экстрагентов - ограниченная растворимость продуктов экстракции РЗМ в органической фазе. Нами установлено, что эффективной сольватирующей добавкой [5], исключающей образование третьей фазы, для бинарных экстрагентов служит нитрат $T O A\left(N R_{3} H N O_{3}\right)$. Однако указанное соединение является продуктом экстракции по механизму бинарной экстракции, а также может экстрагировать нитраты лантаноидов в виде нейтральных комплексов по сольватационному механизму [7]. Поэтому основная цель исследований, представленных в статье, - изучение особенностей экстракции нитратов РЗМ в системах: бинарный экстрагент EHEHPA/ TOA плюс сольватирущая добавка - нитрат TOA; бинарный экстрагент DEHPA/TOA плюс $\mathrm{NR}_{3} \mathrm{HNO}_{3}$. 


\section{Материалы и методы}

Ди-(2-этилгексил) фосфорная кислота (торговые названия Д2ЭГФК, DEHPA, P 204, TOPS 99). 2-этилгексил-моно-2-этилгексил эфир фосфоновой кислоты (торговые названия EHEHPA, PC-88A, P-507, Ionquest 801) $[8,9]$.

Для приготовления бинарных экстрагентов точные навески DEHPA или EHEHPA и $\mathrm{NR}{ }_{3} \mathrm{H}$ $\mathrm{Cl}$ (взятые в стехиометрическом соотношении) растворяли в органическом разбавителе (80\% полного объема). Органическую фазу однократно промывали раствором аммиака (отношение объемов фаз 1:0,9) с концентрацией, равной концентрации DEHPA или EHEHPA. Затем органическую фазу доводили до полного необходимого объема. Затем экстрагент семикратно обрабатывали равными объемами раствора $\mathrm{NH}_{3} 0.001 \mathrm{M}$ до $\mathrm{pH}$ водной фазы $\geq 7$ и до отсутствия $\mathrm{Cl}^{-}$в реэкстрактах. Далее трехкратно промывали водой. Точные содержания DEHPA или EHEHPA и $\mathrm{NR} \mathrm{R}_{3} \mathrm{HCl}$ в исходных реагентах определяли методами алкалиметрического и аргентометрического титрования.

Для приготовления бинарных экстрагентов с добавкой $\mathrm{NR}_{3} \mathrm{HNO}_{3}$ смешивали рассчитанные объемы раствора с известной концентрацией $\mathrm{NR}_{3} \mathrm{HNO}_{3}$ и раствора бинарного экстрагента $\left(\mathrm{NR}{ }_{3} \mathrm{HA}\right)$.

Растворы $\mathrm{Nd}$ (III) готовили растворением соответствующего оксида РЗЭ $(99,9$ \%) в концентрированной азотной кислоте, выпаривали и разбавляли дистиллированной водой.

Соль $N R_{3} H C l$ получали в виде белого твердого вещества из гептанового раствора $N R_{3}$ и водного раствора $\mathrm{HCl}[10]$. Растворы $\mathrm{NR}_{3} \mathrm{HNO}_{3}$ получали 3-кратной обработкой растворов $\mathrm{NR} \mathrm{R}_{3} \mathrm{HCl}$ в органическом разбавителе, водными растворами $4 \mathrm{M} \mathrm{NaNO}$.

Спектрофотометрические измерения проводили с использованием волоконно-оптического спектрометра AvaSpec-ULS2048L. Концентрации элементов в водных фазах анализировали масс-спектрометрией с индуктивно-связанной плазмой с помощью ICP-MS Agilent 7500A. pH растворов определяли потенциометрически рН-метром «Эксперт-001».

\section{Результаты и обсуждение}

Бинарная экстракция нитратов лантаноидов $\left(\operatorname{Ln}\left(N O_{3}\right)_{3}\right)$ может быть описана следующим уравнением (1) основной гетерогенной реакции (без учета специфических взаимодействий в органической фазе, в частности, процессов сольватации):

$$
\mathrm{Ln}_{(\mathrm{B})}^{3+}+3 \mathrm{NO}_{3(\mathrm{(b)}}^{-}+3 N R_{x} H_{(4-x)} A_{(o)} \leftrightarrows \operatorname{LnA}_{3(o)}+3 N R_{x} H_{(4-x)} N O_{3(o)}
$$

где $R_{x} N H_{(4-x)} A$ - бинарный экстрагент (соль, образованная амином $R_{x} N H_{(3-x)}$ и органической кислотой НА); символы (о) и (в) обозначают принадлежность компонентов к органической и водной фазам соответственно. Переход катиона и аниона экстрагируемой неорганической соли является сопряженным и обратимым, соответственно, реэкстракция может быть проведена водой. Отмеченная особенность бинарной экстракции обеспечивает возможность создания новых, экономически эффективных технологий разделения РЗМ. Например, в противоточных экстракционных каскадах на участках экстракции и реэкстракции возможно на порядок уменьшить расход минеральных оснований и кислот.

Форма изотерм экстракции нитратов лантаноидов легкой и средней групп на примеpe $N d\left(N O_{3}\right)_{3}$ (рис. 1) из нейтральных водных растворов растворами $E H E H P A / T O A\left(N R_{3} H A\right)$ в 


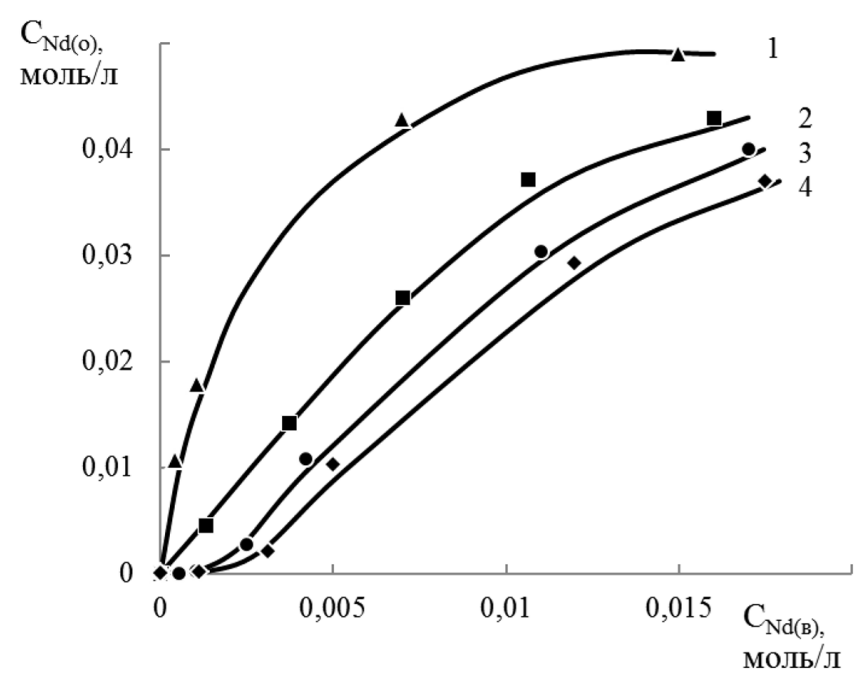

Рис. 1. Изотермы экстракции $N d\left(N_{3}\right)_{3}$ бинарными экстрагентами на основе $E H E H P A$ и TOA, содержащими различную концентрацию нитрата три-н-октиламина. С $\left(N R_{3} H A\right)$ 0,2 моль/л, pH 4,3. Разбавитель толуол. Точки - экспериментальные данные, кривые - модельные данные: $1-\mathrm{C}\left(\mathrm{NaNO}_{3}\right)_{\text {(в) }} 0,01$ моль/л; 2 - $\mathrm{C}\left(\mathrm{NR}_{3} H N \mathrm{H}_{3}\right)_{(\text {о) }} 0$ моль/л; $3-\mathrm{C}\left(\mathrm{NR}_{3} \mathrm{HNO}_{3}\right)_{\text {(о) }} 0,055$ моль/л; $4-\mathrm{C}\left(\mathrm{NR}_{3} H N O_{3}\right)_{\text {(о) }} 0,105$ моль/л

Fig. 1. Isotherms of $\mathrm{Nd}\left(\mathrm{NO}_{3}\right)_{3}$ extraction with binary extractants based on EHEHPA and TOA containing different concentrations of tri-n-octylamine nitrate. $\mathrm{C}\left(N R_{3} H A\right) 0,2 \mathrm{~mol} / \mathrm{l}, \mathrm{pH} 4,3$. Diluent is toluene. Points represent experimental data, while curves correspond to model data. $1-\mathrm{C}\left(\mathrm{NaNO}_{3}\right)_{(\mathrm{B})} 0,01 \mathrm{~mol} / \mathrm{l} ; 2-\mathrm{C}\left(\mathrm{NR}_{3} \mathrm{HNO}_{3}\right)_{(\mathrm{o})} 0 \mathrm{~mol} / \mathrm{l}$; $3-\mathrm{C}\left(\mathrm{NR}_{3} \mathrm{HNO}_{3}\right)_{(\mathrm{o})} 0,055 \mathrm{~mol} / 1 ; 4-\mathrm{C}\left(\mathrm{NR}_{3} \mathrm{HNO}_{3}\right)_{(\mathrm{o})} 0,105 \mathrm{~mol} / 1$

органическом разбавителе качественно соответствует процессу излечения неорганической соли по механизму бинарной экстракции (уравнение 1). Изотермы экстракции линейны (рис. 1, кривая 2) на начальном участке (в области большого избытка экстрагента, при отсутствии высаливающих добавок в водной и органической фазе). Введение в водную или органическую фазу исходных или конечных продуктов реакции (высаливающих добавок) смещает химическое равновесие. Для экстракционной системы $N d\left(N_{3}\right)_{3}-N R_{3} H A$ наблюдаются: значительное повышение $D_{N d}$ при введении одноименного аниона $\left(\mathrm{NO}_{3}^{-}\right.$, натриевая соль) в водную фазу (рис. 1 , кривая 1 ); уменьшение $D_{N d}$ при введении $N R_{3} H N O_{3}$ в органическую фазу (рис. 1, кривые 3, 4).

При бинарной экстракции солей рН водной фазы изменяется мало (в отличие от катионообменной). Для исходного водного раствора 0,01 моль/л $N d\left(N_{3}\right)_{3}$ начальное значение рН 5,2, для равновесных растворов, соответствующих изотермам (рис. 1), в диапазоне 4,2-4,3. Для би-

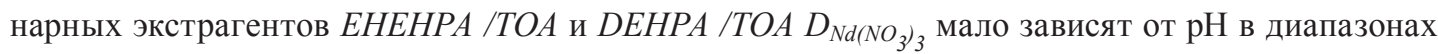
4,6 - 6,2 и 4,0 - 6,2 соответственно. В области больших значений $\mathrm{pH}$ уменьшение $D_{N d}$, повидимому, обусловлено образованием в водной фазе гидроксокомплексов (например, $\mathrm{Nd}(\mathrm{OH})$ $\left.\left(\mathrm{NO}_{3}\right)_{2}\right)$. В области меньших значений $\mathrm{pH}$ происходит конкурирующая экстракция минеральной кислоты (по механизму бинарной экстракции кислот) (уравнения 2, 3):

$$
\begin{aligned}
& H_{(8)}^{+}+\mathrm{NO}_{3(())}^{-}+2 \mathrm{NR}_{3} H A_{(o)} \leftrightarrows \mathrm{NR}_{3} \mathrm{H}\left(\mathrm{HA}_{2}\right)_{(o)}+\mathrm{NR}_{3} \mathrm{HNO}_{3(o)}, \\
& 2 \mathrm{H}_{(())}^{+}+2 \mathrm{NO}_{3(())}^{-}+2 \mathrm{NR}_{3} \mathrm{HA}_{(o)} \leftrightarrows(\mathrm{HA})_{2(o)}+2 \mathrm{NR}_{3} \mathrm{HNO}_{3(o)} .
\end{aligned}
$$


Область рН наиболее резкого изменения $D_{N d}$ соответствует $p K a$ катионообменных экстрагентов. Образующийся в ходе реакции (2) продукт $\left(N R_{3} H\left(H A_{2}\right)\right)$ также может являться бинарным экстрагентом для $\mathrm{Nd}\left(\mathrm{NO}_{3}\right)_{3}$, а $(\mathrm{HA})_{2}$ - катионообменым экстрагентом. Поэтому в сильно кислой области экстракция солей лантаноидов бинарными экстрагентами не отличается от обычной катионообменной экстракции. Однако возможно уменьшение $D_{L n}$ за счет эффектов сольватации $(H A)_{2}$ солями аминов. При низких значениях $D_{L n}$ необходимо учесть параллельную экстракцию неорганических солей по сольватационному механизму (уравнение 4).

$$
\mathrm{Ln}_{(\mathrm{g})}^{3^{+}}+3 \mathrm{NO}_{3(\mathrm{~s})}^{-}+n N R_{3} \mathrm{HNO}_{3(o)} \leftrightarrows\left[\mathrm{Ln}\left(\mathrm{NO}_{3}\right)_{3}\right] \cdot\left(\mathrm{NR}_{3} \mathrm{HNO}_{3}\right)_{n(o)}
$$

При высоких концентрациях $L n$ в органической фазе коэффициент распределения $\left(D_{L n}\right)$ уменьшается - изотерма выходит на плато в область насыщения экстрагента. Оценка предельной концентрации металла в органической фазе ( $\left.{ }^{\text {маx }} C_{L n(o)}\right)$ проведена на основе линейных зависимостей, типичных для области насыщения изотерм:

$$
C_{\operatorname{Ln}(0)}^{-1}=k \cdot C_{L n(\theta)}^{-1}+{ }^{\operatorname{Max}} C_{\operatorname{Ln}(0)}^{-1} \text {. }
$$

Соотношение общей концентрации экстрагента и ${ }^{\text {мax }} C_{L n(o)}$ находится в промежутке от 2,8 до 3,0 , что удовлетворительно согласуется со стехиометрическим коэффициентом 3 при $N R_{3} H A_{(o)}$ для уравнения (1). По-видимому, небольшое превышение содержания $L n$ в органической фазе над стехиометрическим обусловлено дополнительной (параллельной) экстракцией $L n$ по сольватному механизму (уравнение 4).

Для экстракционных систем $\mathrm{Nd}\left(\mathrm{NO}_{3}\right)_{3}-\mathrm{NR}_{3} \mathrm{HNO}_{3}-$ EHEHPA/TOA (DEHPA/TOA) оценка типа комплексов $N d$, образующихся в органической фазе, проведена на основе исследования спектров поглощения $N d^{3+}$ в области 550-620 нм, соответствующей «гиперчувствительному» (к координационному окружению металла) электронному переходу ${ }^{4} I_{9 / 2} \rightarrow{ }^{2,4} G_{7 / 2 ; 5 / 2}$. При концентрации $N d$ в органической фазе, соответствующей линейным участкам изотерм, форма полос поглощения $N d^{3+}$ для систем $N d\left(N O_{3}\right)_{3}-N R_{3} H A-N R_{3} H N O_{3}$ качественно повторяет спектры поглощения $\mathrm{Nd}\left(\mathrm{H} \mathrm{A}_{2}\right)_{3}$ (рис. $2 a, 6$, спектры 3, 4).

Нейтральные комплексы $N d^{3+}$ с различными анионными фосфорсодержащими лигандами (DEHPA, EHEHPA и др.) характеризуются октаэдрической координацией и идентичными спектрами поглощения. В экстракционной фазе, содержащей нитрат триоктиламина (без EHEHPA), нитратные комплексы неодима $\left.\left(\left[\mathrm{Nd}\left(\mathrm{NO}_{3}\right)_{3}\right] \cdot\left(\mathrm{NR}_{3} \mathrm{HNO}_{3}\right)_{n}\right)\right)$ имеют другой вид спектра поглощения (рис. 2б), спектр 1) [7]. Этот спектр очень похож на спектр нейтрального комплекса нитрата неодима с трибутилфосфатом - $\left[N d\left(N_{3}\right)_{3}\right] \times Т Б \Phi_{(o)}($ рис. 26), спектр 2). Таким образом, для исследованных смешанных бинарных экстрагентов не происходит координация $\mathrm{Nd}^{3+} \mathrm{c}$ нитрат-ионом. Обнаруженные эффекты значительного повышения растворимости $\operatorname{Ln} A_{3(o)}$ (до 0,5 моль/л) в присутствии $\left(N R_{3} H N O_{3}\right)$, по-видимому, связаны с сольватационными взаимодействиями и внешнесферной координацией.

Для рассматриваемых экстракционных систем характерен S-образный вид изотерм межфазного распределения РЗМ. Поэтому стандартные графические методы для описания и расчета экстракции одновременно нескольких элементов (рис. 3) неудобны (в отличие от систем с линейными изотермами).

$$
-367-
$$




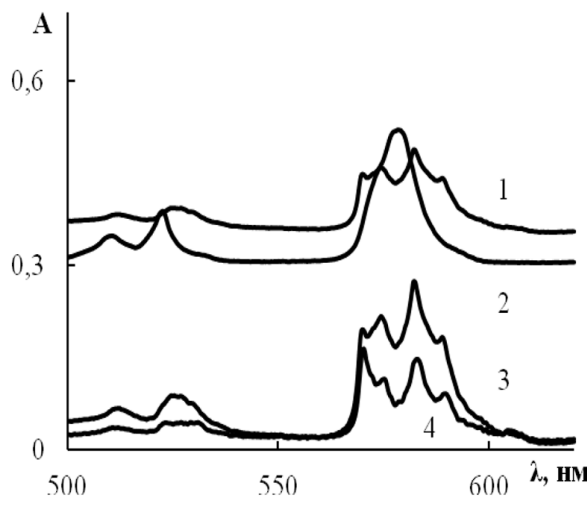

A)

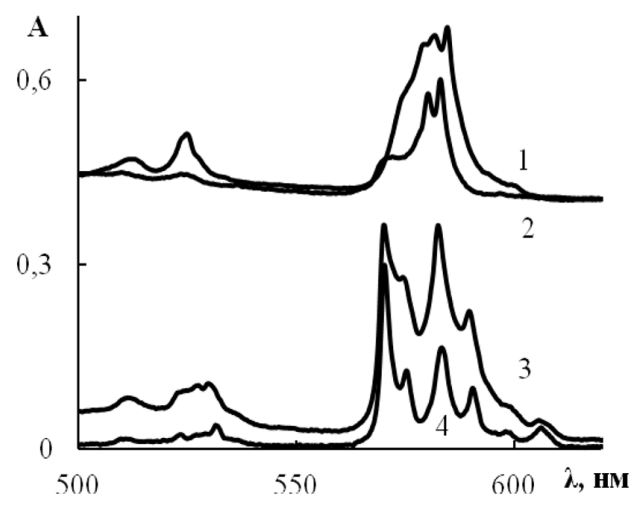

Б)

Рис. 2. Спектры поглощения соединений $N d^{3+}$ : А) $1-\mathrm{C}\left(N d\left(N O_{3}\right)_{3}\right)_{(8)} 0,04$ моль/л; $2-\mathrm{C}\left(N d\left(N O_{3}\right)_{3}\right)_{(8)}$ 0,02 моль/л + $\mathrm{C}\left(\mathrm{NaNO}_{3}\right)_{(8)} 6$ моль/л; $3-\mathrm{C}(H D E H P / T O A)$ 0,2 моль/л, $\mathrm{C}\left(N R_{3} H N O_{3}\right)_{(o)} 0,1$ моль/л; $4-\mathrm{C}(H D E H P)$ (o) 0,2 моль/л; Б) $1-\mathrm{C}\left(N R_{3} H N O_{3}\right)_{(o)} 0,5$ моль/л; $2-50 \%$ ТБФ (о); $3-\mathrm{C}(E H E H P A / T O A)_{(o)} 0,2$ моль/л, $\mathrm{C}\left(N R_{3} H N O_{3}\right)$ (o) 0,1 моль/л; $4-\mathrm{C}(E H E H P A)_{(o)} 1$ моль/л. Разбавитель - толуол

Fig. 2. Absorption spectra of extracted $N d$ complex: A) $1-\mathrm{C}\left(N d\left(N O_{3}\right)_{3}\right)_{(6)} 0,04 \mathrm{~mol} / 1 ; 2-\mathrm{C}\left(N d\left(N O_{3}\right)_{3}\right)_{(8)} 0,02 \mathrm{~mol} / 1$ л + $+\mathrm{C}\left(\mathrm{NaNO}_{3}\right)_{(\mathrm{g})} 6 \mathrm{~mol} / \mathrm{l} ; 3-\mathrm{C}(H D E H P / T O A) \quad 0,2 \mathrm{~mol} / \mathrm{l}, \mathrm{C}\left(\mathrm{NR}_{3} H_{\left.N N O_{3}\right)_{(o)}} 0,1 \mathrm{~mol} / \mathrm{l} ; 4-\mathrm{C}(H D E H P)_{(o)} 0,2 \mathrm{~mol} / \mathrm{l}\right.$; Б) $1-\mathrm{C}\left(\mathrm{NR}_{3} \mathrm{HNO}_{3}\right)_{(o)} 0,5 \mathrm{~mol} / \mathrm{l} ; 2-50 \% \mathrm{TBP}(\mathrm{o}) ; 3-\mathrm{C}(E H E H P A / T O A)_{(o)} 0,2 \mathrm{~mol} / \mathrm{l}, \mathrm{C}\left(\mathrm{NR}_{3} H N O_{3}\right)_{(o)} 0,1 \mathrm{~mol} / \mathrm{l}$; $4-\mathrm{C}(E H E H P A)_{(o)} 1 \mathrm{~mol} / 1$. Diluent is toluene

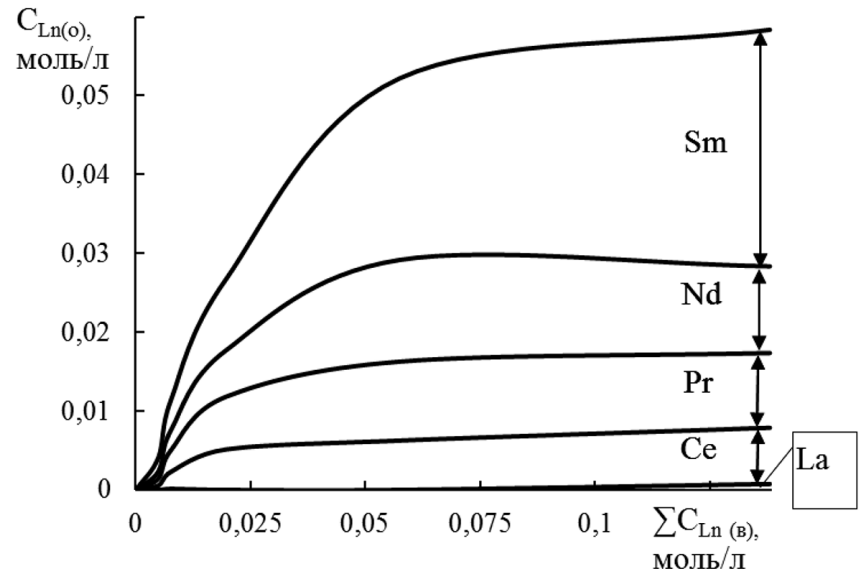

Рис. 3. Изотерма совместного извлечения пяти нитратов РЗМ бинарным экстрагентом на основе EHEHPA, TOA $\mathrm{NR}_{3} \mathrm{HNO}_{3}$. C $(E H E H P A / T O A)_{(\text {о) }} 0,2$ моль/л, С $\left(N R_{3} H N O_{3}\right)_{(\text {о) }} 0,105$ моль/л, разбавитель толуол, $\mathrm{pH} 5,0, \mathrm{O}: \mathrm{B}=1$. Исходные концентрации пяти металлов в водной фазе равны

Fig. 3. Isotherm of the joint extraction of five rare-earth nitrates with binary extractant based on EHEHPA, TOA, $\mathrm{NR}_{3} \mathrm{HNO}_{3} \cdot \mathrm{pH}=5 . \mathrm{O}: \mathrm{B}=1$. Diluent is toluene. The initial concentrations of five metals in the aqueous phase are equal

Для количественного описания процесса бинарной экстракции может быть применен численный метод с решением системы нелинейных уравнений, представляющих равновесия с участием различных компонентов в гетерогенной системе. При этом необходимо учитывать дополнительные значимые взаимодействия в органической фазе, не включенные в уравнение (1). Соответственно, важно выбрать компоненты органической фазы, 
которые преимущественно существуют в системе в широком диапазоне составов и концентраций, минимизировав набор значимых равновесных процессов (и констант их характеризующих).

На основании математического моделирования набора экспериментальных изотерм (для нитратов средних и легких РЗМ) установлено, что корректное описание экстракционных систем $\mathrm{Ln}\left(\mathrm{NO}_{3}\right)_{3} \mathrm{NR}_{3} \mathrm{HA}-\mathrm{NR}_{3} \mathrm{HNO}_{3}$ возможно при учете дополнительных взаимодействий: сольватации $L n A_{3(o)}$ нитратом амина и самоассоциации $N_{3} H N O_{3}$. С учетом процессов сольватации уравнение бинарной экстракции можно записать в виде

$$
\mathrm{Ln}_{(\mathrm{B})}^{3^{+}}+3 \mathrm{NO}_{3(\mathrm{~s})}^{-}+3 N R_{3} H A_{(o)} \leftrightarrows \operatorname{LnA}_{3}\left(\mathrm{NR}_{3} H N O_{3}\right)_{s(o)}+(3-n s) N R_{3} H N O_{3(o)},
$$

где $n s$ - число сольватации. Для количественного описания экстракционных систем предложено уравнение концентрационной константы бинарной экстракции $\left(K_{b e x}\right)$ :

$$
\begin{aligned}
& K_{b e x}=\left(\left[\operatorname{Ln}_{(6)}^{3+}\right] \cdot\left[N O_{3(s)}^{-}\right]^{3} \cdot \gamma_{ \pm}^{4} \cdot\left[N R_{3} H A_{(o)}\right]^{3}\right)^{-1} \cdot\left[\operatorname{LnA}_{3}\left(N R_{3} H N O_{3}\right)_{n s(o)}\right] \cdot \\
& {\left[N_{3} R_{3 N O} O_{3(o)}\right]^{(3-n s)}}
\end{aligned}
$$

где $\gamma_{ \pm}$- среднеионный коэффициент активности $\operatorname{Ln}\left(N O_{3}\right)_{3}$ в водной фазе (коэффициенты активности компонентов органической фазы включены в концентрационную константу). Используя константу димеризации $\left(K_{d i m}\right)$, возможно получить уравнение, связывающее равновесную и общую концентрации нитрата амина в органической фазе:

$$
\left[N R_{3} H_{N O}(o)\right]=0.25 \cdot K_{\operatorname{dim}^{-1}}{ }^{-1}\left(\left(1+8 \cdot K_{d i m} \cdot C\left(N R_{3} H N O_{3(o)}\right)\right)^{0.5}-1\right) .
$$

Для уточнения значений $K_{b e x(L n)}, K_{d i m}$, и $n s$ для набора экспериментальных изотерм экстракции нитратов различных РЗМ минимизировали сумму квадратов разницы измеренных и расчетных значений коэффициентов распределения $\left(\sum\left({ }^{e x p} D_{L n}-{ }^{c a l c} D_{L n}\right)^{2}\right)$. При допущении, что в ряду РЗМ легкой и средней групп постоянны $K_{d i m}=87,3$ и $n s=1$. Пример модельных изотерм приведен на рис. $1, K_{b e x(L n)}-$ в табл. 1.

Небольшие различия экспериментальных и расчетных значений $D_{L n}$ наблюдаются в области насыщения изотерм. По-видимому, это связано с дополнительными механизмами экстракции (уравнение 4). Тем не менее, по нашему мнению, полученное описание изотерм для экстракционных систем $\operatorname{Ln}\left(\mathrm{NO}_{3}\right)_{3}-\mathrm{NR}_{3} \mathrm{HA}-\mathrm{NR}_{3} H N O_{3}$ позволяет моделировать поведение $\mathrm{Ln}$ для последующих расчетов противоточных экстракционных каскадов.

Таблица 1. Коэффициенты разделения $\left(\beta_{L n / N d}\right)$ и концентрационные константы бинарной экстракции

\begin{tabular}{|c|c|c|c|c|c|c|c|c|c|c|}
\hline & & $\mathrm{La}$ & $\mathrm{Ce}$ & $\operatorname{Pr}$ & $\mathrm{Nd}$ & $\mathrm{Sm}$ & $\mathrm{Eu}$ & $\mathrm{Gd}$ & $\mathrm{Tb}$ & Dy \\
\hline \multirow{2}{*}{$\begin{array}{l}\text { EHEHPA/ } \\
\mathrm{TOA} \mathrm{NR}_{3} \mathrm{HNO}_{3}\end{array}$} & $\beta_{\text {Ln/Nd }}$ & 0,052 & 0,44 & 0,92 & 1,00 & 3,55 & 4,56 & 5,24 & 9,85 & 14,7 \\
\hline & $\operatorname{LgK}_{\text {bexLn }}$ & $-0,62$ & 3,08 & 4,38 & 4,51 & 6,71 & 7,15 & 7,39 & 8,49 & 9,18 \\
\hline \multirow{2}{*}{$\begin{array}{l}\text { DEHPA/ } \\
\text { TOA NR }{ }_{3} \mathrm{HNO}_{3}\end{array}$} & $\beta_{\mathrm{Ln} / \mathrm{Nd}}$ & 0,078 & 0,39 & 0,83 & 1,00 & 6,79 & 6,98 & 7,03 & 8,71 & 13,4 \\
\hline & $\operatorname{LgK}_{\text {bexLn }}$ & $-0,53$ & 2,27 & 3,10 & 3,91 & 7,24 & 7,29 & 7,30 & 7,67 & 8,42 \\
\hline
\end{tabular}
$\left(L g K_{b e x L n}\right)$ нитратов в ряду РЗМ. Разбавитель - толуол

Table 1. Separation factors $\left(\beta_{L n / N d}\right)$ and concentration constants of binary extraction $\left(\operatorname{Lg} K_{b e x L n}\right)$ of nitrates in the series of rare-earth metals. Diluent is toluene 
Таким образом, для экстракционных систем $\operatorname{Ln}\left(\mathrm{NO}_{3}\right)_{3}-E H E H P A / T O A-N_{3} \mathrm{HNO}_{3}$ и $L n\left(N O_{3}\right)_{3}-D E H P A / T O A-N R_{3} H N O_{3}$, где $L n-L a \ldots D y$, разработано модельное описание, позволяющее в широком диапазоне составов и концентраций компонентов рассчитывать коэффициенты распределения и разделения РЗМ. Полученное количественное описание условий экстракции-реэкстракции РЗМ в смесях является базисом для расчета противоточных экстракционных каскадов их разделения и очистки с применением бинарных экстрагентов.

\section{Выводы}

Исследована экстракция нитратов РЗМ смесями экстрагентов - бинарных (на основе фосфорсодержащих кислот и третичного амина) и нейтральных (на основе нитрата аминов). Показано, что в подобных смешанных экстрагентах нитрат амина выступает не только как сольватирующая, но и как высаливающая добавка. Установлено, что дополнительные взаимодействия в органической фазе незначительно снижают селективность извлечения РЗМ. При этом за счет эффекта сольватации значительно повышается растворимость металлов в органической фазе, что является условием применимости экстрагентов для промышленного разделения РЗМ. Эффект высаливания понижает коэффициенты распределения металлов, что позволяет проводить их реэкстракцию водой и, в свою очередь, позволит значительно сократить расход минеральных кислот и оснований в экстракционных противоточных каскадах при разделении РЗМ.

\section{Благодарности / Acknowledgements}

Исследования выполнены в рамках базового проекта V.46.1.1.

The studies were carried out as part of the base project V.46.1.1.

\section{Список литературы / References}

1. Холькин А.И., Кузьмин В.И., Пашков Г.Л. и др. Бинарная экстракции и перспективы ее применения. Изв. Сиб. отд. АН СССР. Сер. хим. наук 1990. Вып. 5. С. 3-17. [Khol'kin A.I., Kuz'min V.I., Pashkov G.L. et al. Binary extraction and perspectives for its application. Math. Sib. Dep. USSR Academy of Sciences. Ser. chem. Sciences 1990. (5), P. 3-17. (In Russ.)]

2. Belova V.V., Voshkin A.A., Khol'kin A.I., Payrtman A.K. Solvent extraction of some lanthanides from chloride and nitrate solutions by binary extractants, Hydrometallurgy 2009. Vol. 97, P. 198-203.

3. Belova V.V., Khol'kin A.I. Extraction of mineral acids and lanthanum salts with binary extractants. Russ. J. Inorg. Chem. 2015. Vol. 60, P. 1157-1162.

4. Kalyakin S.N., Kuz'min V.I., Mulagaleeva M.A. Binary extraction of lanthanide(III) chlorides using carboxylates and dialkylphosphates of secondary and tertiary amines. Hydrometallurgy 2015. Vol. 151, P. 116-121.

5. Kalyakin S.N., Kuzmin V.I., Mulagaleeva M.A. Binary extraction of neodymium nitrate using 2-ethylhexylphosphonic acid 2-ethylhexyl mono ester and tri-n-octylamine. Journal of Molecular Liquids 2019. Vol. 273, P. 45-49.

6. Калякин С.Н., Кузьмин В.И., Мулагалеева М.А. Извлечение нитратов лантаноидов (III) смесями бинарных экстрагентов - карбоксилатов и диалкилфосфатов аминов. Журнал СФУ. Химия 2015. T. 8(4), C. 580-589. [Kalyakin S.N., Kuzmina V.I., Mulagaleeva M.A. Solvent Extrac- 
tion the Nitrate of Lanthanide (III) Binary Mixtures Extragents - Carboxylate and Dialkyl Phosphate Amines. Journal of Siberian Federal University. Chemistry 2015. Vol. 8 (4), P. $580-589$ (in Russ.)]

7. Quinn J.E., Soldenhoff K.H., Stevens G.W. Solvent Extraction of Rare Earth Elements Using a Bifunctional Ionic Liquid. Part 2: Separation of Rare Earth Elements. Hydrometallurgy 2017. Vol. 169, $621-628$.

8. Li D., Bai Y., Sun X., Liu S., in: I.M. London, Goode J.R., Moldoveanu G., Rayat M.S. (Eds.), Advances in solvent extraction and separation of rare earths. $52^{\text {nd }}$ Conference of Metallurgists (COM). Canadian Institute of Mining Metallurgy and Petroleum, Montreal, Canada 2013. P. 367-374.

9. Khaironie M.T., Masturah M., Meor Yusoff M.S., Nazaratul Ashifa S. Solvent Extraction of Light Rare Earth Ions Using D2EHPA from Nitric Acid and Sulphuric Acid Solutions. Advanced Materials Research 2014. Vol. 970, P. 209-213.

10. Katsuta Sh., Yoshimoto Y., Okai M., Takeda Ya., Bessho K. Selective Extraction of Palladium and Platinum from Hydrochloric Acid Solutions by Trioctylammonium-Based Mixed Ionic Liquids. Ind. Eng. Chem. Res. 2011. Vol. 50, P. 12735-12740. 